PSYCHOMETRIKA-VOL. 78, NO. 3, 395

JULY 2013

DOI: $10.1007 / \mathrm{s} 11336-013-9344-2$

\title{
ANNOUNCEMENT RE: A SURVEY OF THE SOURCES OF NOISE IN FMRI DOI 10.1007/S11336-012-9294-0
}

This article was intended to be the first article in the special ARCS section on fMRI, published in v78n2. It was accidentally omitted due to an error in the production process, and is therefore being printed in $\mathrm{v} 78 \mathrm{n} 3$ instead.

MANAGING EDITOR

Gwen Exner 